\title{
THE TECHNIQUE OF MEASURING THE HEIGHT IN PATIENTS WITH OSTEOPOROSIS
}

\author{
Kottahachchi DC 1,2, Gao F', Topliss DI¹, Sztal-Mazer S1. \\ ${ }^{1}$ The Alfred Diabetes and Endocrinology Department, Melbourne, Australia. ${ }^{2}$ The Faculty of Medicine, the University of Kelaniya, \\ Sri Lanka.
}

\section{ABSTRACT}

Introduction: In patients with osteoporosis, the loss of height is indicative of vertebral fractures and periodic height measurement is an essential component of their clinical assessment. It is a challenging task in these patients and there is no recommended, standard technique for measuring the height of these patients. Our objective was to assess the accuracy of the stadiometer and the drop-down scale with the carpenter's level for height measurements.

Method: Height measurements were taken from 30 consecutive patients using the stadiometer and the drop-down scale with carpenter's scales. Again, three height measurements were taken from 172 patients using only the drop-down scale with carpenter's scale.

Results: The height measurements from stadiometer gave more accurate results compared to drop-down scale $(\mathrm{p}<0.001)$. There was no statistically significant difference between a single height measurement and the average of three height measurements.

Conclusions: The stadiometer with a carpenter's level can provide accurate height measurements in patients with osteoporosis. The accuracy of a single measurement is as good as the average of three height measurements.

Running title: The height measurements in osteoporosis

Keywords: Osteoporosis, stadiometer, drop-down scale, carpenter's level, height measurements.

\section{INTRODUCTION}

Osteoporosis $(\mathrm{OP})$ is becoming a major health related issue worldwide, so as in Australia. According to the Geelong Osteoporosis study, the prevalence of OP is $6 \%$ among males and $23 \%$ among females over the age of 50 years (1). Due to the diversity of the patients to the clinical practice, managing patients with osteoporosis has always been a challenge to the physicians. Approximately $2 / 3$ of the vertebral fractures are asymptomatic (2) and proper assessment can predict the risk of further fractures and morbidity to these patients (3). During the management of these patients, in the process of decision making, many clinical parameters are taken into consideration and the height loss of the patient is one of the important parameters considered, as it can indicate vertebral fractures. According to the International Society of Clinical Densitometry, women who have had a height loss of $\geq 1.6$ inches $(4 \mathrm{~cm})$ from their peak height, men who have had a height loss of $\geq 2.4(6 \mathrm{~cm})$, or patients with prospective height loss of $>0.8$ inches $(2 \mathrm{~cm})$ in women or $>1.2$ inches $(3 \mathrm{~cm})$ in men, warrant vertebral fracture assessment by DEXA technology (4).

Height is an easy clinical parameter to measure. However, it can be one of the most challenging tasks in patients with OP. Although accurate measurement of height in OP patients has been appreciated, the correct technique has not been studied extensively, especially to make recommendations to busy OP clinic setup. Osteoporosis society Canada has published an update in 2005 regarding the correct technique in measuring height in OP patients (5). They have recommended using a carpenter's level in order to obtain accurate height measurements. Measuring height with the stadiometer (Figure 1) and the dropdown scale (Figure 2) are the standard methods that are being used to measure the heights of the patients with OP. The objective of our study was to compare the standard method of measuring height with the stadiometer with a carpenter's level and by the drop-down scale with a carpenter's level. Considering the difficulty of doing several measurements in busy osteoporosis clinics, we also wanted to check whether the accuracy of the average of three measurements is as good as a single measurement.

\section{METHOD}

We measured height in 30 consecutive patients who attended 


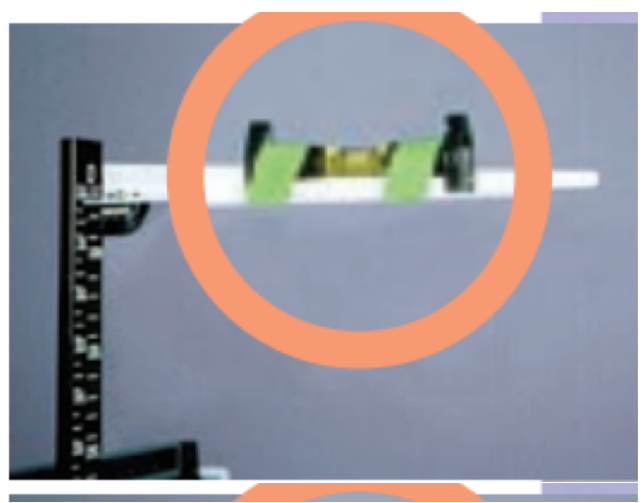

Figure 1. Stadiometer with the carpenter's level

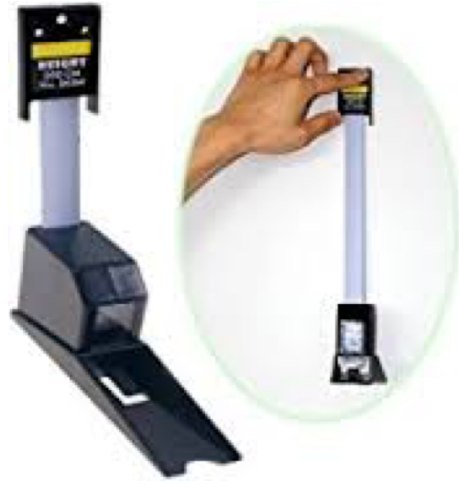

Figure 2. Drop-down scale the OP clinic using both the stadiometer with carpenter's level and the drop-down scale with carpenter's level keeping with the technique mentioned below. In addition, we drew two parallel lines on either side of the drop-down scale to make the patient stand straight as possible. Height was measured three times and the average was taken. We also measured height three times in 172 OP patients only by using the drop-down scale with the carpenter's level to see whether one height measurement is as good as the average of three measurements.

The technique used in our clinic to measure heights was as follows. The patient was asked to stand straight with heels together with the feet angled at about $60^{\circ}$, in bare feet or with stockings. When using the drop-down scale, the heels were kept touching the wall or the heel plate. When the stadiometer was used, the buttocks or back were kept touching the wall or the unit. The patient was asked look straight ahead and the head was positioned in the "Frankfort plane" where the inferior border of the bony orbit is in line with the groove at the top of the tragus of the ear (Figure 3). Patients were asked to actively stretch to a fully erect position while keeping their feet flat on the floor. The horizontal arm of the measuring unit was gently pulled down till it compresses the hair as much as possible, and till it remains at a rigid right angle to the measuring scale. The performance of the measuring rod on the platform weight scale was enhanced by attaching a small carpenter's level to the horizontal arm to make sure it remains in the desired position (Figure 1). Height was recorded to the closest $\mathrm{mm}$. The measurement was repeated three times, and the average was calculated to the closest $\mathrm{mm}$. Patients were asked to step away from the device and they were repositioned between measurements.

\section{RESULTS}

Thirty OP patients (Females =16) with an average age of $56.4 \pm 14.6$ years had their heights measured by both methods. The results showed that there is a statistically significant difference between the height measurement from the stadiometer and the drop-down scale in the 30 patients with a p-value of 0.00 ( $\mathrm{p}<0.001$ ), indicating the stadiometer measurement was more accurate than the drop-down scale measurement. Among the 172 patients who had their height measured three times, there was no statistical difference between the first value and the average value $(p=0.377)$ or among the three measured height values. Therefore, measuring a single height value was found to be accurate and adequate.

\section{RESULTS}

Although height measurement is a very useful, simple, and a cost-effective tool in detecting vertebral fractures in $\mathrm{Op}$ patients, it's overlooked by many physicians. Obtaining an accurate measurement of height in OP patients is a challenge, as many patients are elderly and frail. The correct technique of measuring height in $\mathrm{OP}$ patients has not been studied extensively and this study gives a certain amount of useful information with regard to this aspect. Our study reiterated the accuracy of stadiometer for measuring height in OP patients and the use of stadiometer with the carpenter's level with the correct technique can ensure more accurate results.

Osteoporosis is usually a condition of elderly, where mobility is a problem. This becomes even more difficult especially for patients with vertebral fractures. Taking several height measurements in a patient of this nature is difficult in busy outpatient settings. Our study demonstrated that a single height measurement was as

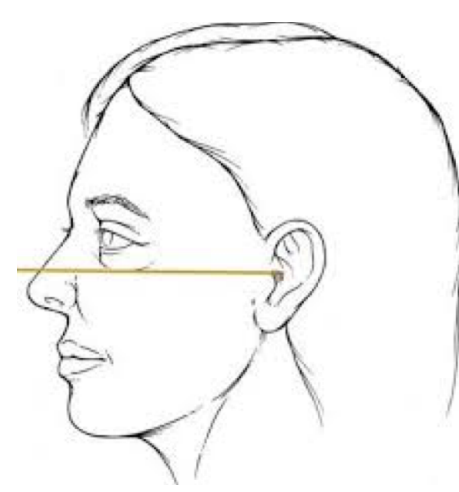

Figure 3. Frankfort plane 
good as the average of three height measurements. Therefore, the ability to obtain an accurate height with one measurement is of particular advantage in busy outpatient clinics especially for elderly patients with OP.

\section{CONCLUSION}

Provided the correct technique is used, the stadiometer can provide accurate height measurements in patients with osteoporosis. A single height measurement can give accurate results and can be recommended for the assessment of patients with osteoporosis. This useful information can be used to measure the height and to compare with the previously documented heights in detecting vertebral fractures.

\section{REFERRENCES}

1. Henry MJ, Pasco JA, Nicholson GC, Kotowicz MA. Prevalence of osteoporosis in Australian men and women: Geelong Osteoporosis Study. Medical journal of Australia 2011; 195(6): 321-322.

2. Fink HA, Milavetz DL, Palermo L, Nevitt MC, Cauley JA, Genant HK, et al. What proportion of incident radiographic vertebral deformities is clinically diagnosed and vice versa? Journal of Bone Mineral Research 2005; 20(7): 1216-1222.

3. $\mathrm{Xu}, \mathrm{W}$ anWan et al. Height loss, vertebral fractures, and the misclassification of osteoporosis. Bone 2011; 48(2): 307-311.

4. Baim S, Binkley $\mathrm{N}$, Bilezikian JP, Kendler DL, Hans DB, Lewiecki EM, et al. Official Positions of the International Society for Clinical Densitometry and executive summary of the 2007 ISCD Position Development Conference. Journal of Clinical Densitometry 2008; 11(1): 75-91.

5. Kerry Siminoski. Tools and techniques. Accurate height assessment to detect hidden vertebral fractures. Osteoporosis update 2005; 9(2): 4-5. 